\title{
Effect Observation of Clinical Treatment with Oxaliplatin and Tiggio for Biliary Tract Tumors on Advanced Stage
}

\author{
Wei Zheng, Haibin Wang, Jiao Zhou* \\ Shaanxi Provincial People's Hospital, Xi'an 710068, Shaanxi Province, China
}

\begin{abstract}
[Abstract] Objective: To investigate the effect of oxaliplatin combined with tiggio in the treatment of advanced biliary tract tumors. Methods: The research period was from November 2019 to November 2020. 80 patients with advanced biliary tumor disease were enrolled. They were divided into groups according to the order of admission, with 40 cases in each group. The control group received oxaliplatin combined with gemcitabine, and the experimental group received oxaliplatin combined with tiggio. Incidence of adverse reactions, time to disease progression, survival time and clinical efficacy were checked and assessed. Results: Compared with the incidence of adverse reaction of the experimental group, which was $5.00 \%(2 / 40)$, the incidence of adverse reaction of the control group was $25.00 \%$ (10/40). The chi-square value $=6.2745$, $p$-value $=0.0122$. The time to progression and survival time of patients in the experimental group were shorter than those of the control group, with significant differences between the groups $(\mathrm{p}<0.05)$; the clinical efficacy of the experimental group and the control group were $97.50 \%(39 / 40)$ and $77.50 \%(31 / 40)$ respectively, the comparative chi-square value $=7.3143$, p-value $=0.0068$. Conclusion: The combined treatment of oxaliplatin and Tiggio in the treatment of advanced biliary tract tumors has higher safety and reduces the incidence of adverse reactions.
\end{abstract}

Key words: Advanced stage; Biliary tumors; Oxaliplatin; Tiggio

Publication date: May, 2021; Publication online: 31 May, 2021

*Corresponding author: Jiao Zhou, zhengwei03168@163.com

There is no specificity in biliary tract tumors, because it is already advanced biliary tract tumors at the time of diagnosis. In the treatment of advanced biliary tract tumors, it is mainly focused on reducing the patient's body burden, control the incidence of adverse reactions, and improve the quality of life of the patients. Patients with advanced tumors require chemotherapy and radiotherapy ${ }^{[1]}$.

\section{General information and methods}

\subsection{General information}

The research period was from November 2019 to November 2020, and 80 patients with advanced biliary tumors were enrolled. They were grouped according to the order of admission, with 40 cases in each group. Experimental group: There were 23 males and 17 females, the age range was 46-78 years old, and the average age was (62.35 \pm 5.42$)$ years; control group: 21 males, 19 females, age range was
45-78 years old, and the average age was (62.58 \pm 5.32$)$ years; the information in the research were comprehensively sorted and analyzed, and statistical software was used to check the results, and the results were not significantly different $(\mathrm{p}>0.05)$.

\subsection{Methods}

The control group received the treatment of oxaliplatin combined with gemcitabine, and each time $130 \mathrm{mg} / \mathrm{m}^{2}$ of oxaliplatin was administered (manufacturer: Sichuan Meida Kangjiale Pharmaceutical Co., Ltd., approval number: National Medicine Zhunzi H20050141) to the patients via intravenous injection, 2 hours each treatment session, one session a day, and was administered from the first day. $1000 \mathrm{mg} / \mathrm{m}^{2}$ gemcitabine was administered (manufacturer: Lilly France SAS, approval number: National Medicine Zhunzi H20100030) to the patient via intravenous drip 
treatment for half an hour in each session, on the first and eighth days of treatment. The treatment period of the two groups of patients was 21 days, and the treatment was adhered to for 2 weeks.

The experimental group received oxaliplatin combined with Tiggio, and each time $130 \mathrm{mg} / \mathrm{m}^{2}$ oxaliplatin was administered (manufacturer: Sichuan Meida Kangjiale Pharmaceutical Co., Ltd., approval number: National Medicine Zhunzi H20050141) to patients via intravenous drip, 2 hours each session, one session a day, administered from the first day. According to the actual body surface area of the patient, appropriate amount of Tiggio drug (manufacturer: Dapeng Pharmaceutical Industry Co., Ltd., approval number: National Medicine Zhunzi H20100229) was administered for treatment. If the patient's body surface area is under $125 \mathrm{~m}^{2}, 80 \mathrm{mg}$ Tiggio drug was administered to the patients each day; if the patient's body surface area is between $125 \sim 150 \mathrm{~m}^{2}, 100 \mathrm{mg}$ of Tiggio was administered each day; if the patient's body surface area is above $150 \mathrm{~m}^{2}$, $120 \mathrm{mg}$ of Tiggio was administered each day; 2 times a day, administered from day 1 to day 14 of the treatment course.

\subsection{Assessment criteria}

The incidence of adverse reaction, the time to disease progression, survival time and clinical efficacy were checked and assessed.

\section{Statistical analysis}

All data were checked by SPSS20.0 statistical software. Normally distributed measurement data are expressed as (mean \pm standard deviation), and measurement data that meets normal distribution are checked by paired t-test; count data is expressed as (\%) percentage, counting The paired data was checked with chi-square test; the results between the groups were significantly different, and there was statistical significance $(\mathrm{p}<0.05)$.

\section{Results}

\subsection{Assessment on incidence of adverse reactions}

Compared with the incidence of adverse reactions of the experimental group which was $5.00 \%(2 / 40)$, the incidence of adverse reactions of the control group was $25.00 \%$ (10/40) which was higher, and the result chi-square value $=6.2745$ and $\mathrm{p}$-value $=0.0122$. See Table 1 .

Table 1. Assessment on the Incidence of Adverse Reactions [n(\%)]

\begin{tabular}{|c|c|c|c|c|c|c|}
\hline Group & Diarrhea & $\begin{array}{c}\text { Nausea and } \\
\text { Vomiting }\end{array}$ & Anemia & $\begin{array}{c}\text { Peripheral } \\
\text { Neurotoxicity }\end{array}$ & $\begin{array}{c}\text { Liver Function } \\
\text { Damage }\end{array}$ & Incidence \\
\hline $\begin{array}{l}\text { Experimental } \\
\qquad(\mathrm{n}=40)\end{array}$ & $1(2.50 \%)$ & $0(0.00 \%)$ & $0(0.00 \%)$ & $0(0.00 \%)$ & $1(2.50 \%)$ & $2(5.00 \%)$ \\
\hline Control $(n=40)$ & $3(7.50 \%)$ & $1(2.50 \%)$ & $2(5.00 \%)$ & $2(5.00 \%)$ & $2(5.00 \%)$ & $10(25.00 \%)$ \\
\hline $\mathrm{X}^{2}$ & & & & & & 6.2745 \\
\hline $\mathrm{p}$ & & & & & & 0.0122 \\
\hline
\end{tabular}

3.2 Assessment on the time to disease progression and survival time

Compared with the control group, the time to disease progression and survival time of patients in the experimental group were shorter, and the difference between the groups was significant $(\mathrm{p}<0.05)$; see Table 2 .

Table 2. Assessment on the time to disease progression and survival time (months)

\begin{tabular}{ccc}
\hline Group & Time to Disease Progression & Survival Time \\
\hline Experimental $(\mathrm{n}=40)$ & $4.4 \pm 0.23$ & $4.3 \pm 0.23$ \\
Control $(\mathrm{n}=40)$ & $5.2 \pm 1.05$ & $9.7 \pm 1.03$ \\
$\mathrm{t}$ & 4.7071 & 32.3608 \\
$\mathrm{p}$ & 0.0000 & 0.0000 \\
\hline
\end{tabular}

\subsection{Assessment on clinical efficacy}

The clinical efficacy of the experimental group and the control group were $97.50 \%(39 / 40)$ and $77.50 \%(31 / 40)$ respectively, the comparative chi-square value $=7.3143$, $\mathrm{p}$-value $=0.0068$. See Table 3 . 
Table 3. Assessment on clinical efficacy [n(\%)]

\begin{tabular}{ccccc}
\hline Group & Ineffective & Effective & Significantly Effective & Treatment Efficacy \\
\hline Experimental $(\mathrm{n}=40)$ & $1(2.50 \%)$ & $17(42.50 \%)$ & $22(55.00 \%)$ & $39(97.50 \%)$ \\
Control $(\mathrm{n}=40)$ & $9(22.50 \%)$ & $12(30.00 \%)$ & $19(47.50 \%)$ & $31(77.50 \%)$ \\
$\mathrm{X}^{2}$ & & & & 7.3143 \\
$\mathrm{p}$ & & & & 0.0068 \\
\hline
\end{tabular}

\section{Discussion}

Tumors that occur in the biliary system are referred to as biliary tract tumors, which is not only limited to gallbladder tumors, but also include extrahepatic biliary tract tumors. Among them, the incidence of gallbladder tumors is relatively high ${ }^{[2-3]}$. As the clinical symptoms of biliary tract tumors are not specific, patients mainly manifest the symptoms of fever, chills, anorexia, diarrhea, vomiting, and weight loss, etc. Most patients will develop jaundice, but they are almost advanced biliary tract tumors when diagnosed. The prognosis of patients with advanced biliary tract tumors is not good, and prone to recurrence after treatment, which seriously threatens the life safety of patients. During the treatment, the patient's body burden is reduced and the quality of life is improved ${ }^{[4-5]}$.

\section{Conclusion}

Oxaliplatin is a new type of platinum-based anticancer drug with strong activity and low toxicity. The inhibitory effect on bone marrow is lighter, and then hydrated derivatives are formed, which interact with DNA molecules to cause interand intra-chain cross-linking of DNA, which has inhibitory effects on DNA synthesis, and then anti-tumor activity develops, which has inhibitory effects on tumor cells in the body ${ }^{[6-7]}$. Tiggio is a type of fluorouracil derivative, which is converted into 5-FU in the body and has inhibitory effects on tumor cell proliferation. This kind of drug has longer drug effects and obvious anti-tumor effects ${ }^{[8]}$. The combined use of the two drugs can give full play to the synergy between them, has high safety, and is not conducive to cause adverse reactions in patients ${ }^{[8]}$.

In summary, the combined treatment of oxaliplatin and Tiggio in the treatment of advanced biliary tract tumors has higher safety and reduces the incidence of adverse reactions.

\section{References}

[1] Cai WB. Effect of oxaliplatin combined with tiggio on the clinical treatment of 70 cases of advanced biliary tumors [J]. Journal of Mathematical Medicine, 2017, 30(7):1029-1030.

[2] $\mathrm{Mu}$ HC. Treatment efficacy of S-1 combined with oxalipaltin as the first line chemotherapy in patients with advanced biliary tract cancer (BTC) $[\mathrm{J}]$. Electronic Journal of Clinical Medical Literature, 2017, 4(31):6093.

[3] Qin YC. Clinical trial of S-1 combined with oxalipaltin as the first line chemotherapy in patients with advanced biliary tract cancer $[\mathrm{J}]$. China Continuing Medical Education, 2016, 8(26):162-163.

[4] Yao GL. Efficiency and safety of s-1 plus oxaliplatin and gemcitabine plus oxaliplatin in the treatment of advanced bile duct cancer [J]. Journal of Basic and Clinical Oncology, 2018, 31(6):488-491.

[5] Lei HL. Analysis on the clinical efficacy of oxaliplatin combined with s-i in the treatment of advanced biliary tract tumor $[\mathrm{J}]$. Guide of China Medicine, 2016, 14(29):151-152.

[6] Feng XG, Yang R, Yuan B et al. Efficacy and side effects of Oxaliplatin and S-1 in treating advanced biliary tract carcinoma [J]. Journal of Modern Oncology, 2015(20):2988-2989.

[7] Yu JH, Wang L, Yu JY et al. Clinical trial of S-1 combined with oxalipaltin as the first line chemotherapy in patients with advanced biliary tract cancer $[\mathrm{J}]$. Chinese Journal of Geriatric Care, 2014(6):58-61.

[8] Halim A, M, et al. A Phase II study of outpatient biweekly gemcitabine-oxaliplatin in advanced biliary tract carcinomas $[\mathrm{J}]$. Japanese Journal of Clinical Oncology, 2010, 41(2):217-24. 